Cinema Today 



\section{Cinema Today}

A Conversation with Thirty-nine Filmmakers from around the World

ELENA OUMANO

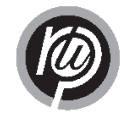

RUTGERS UNIVERSITY PRESS 


\section{LIBRARY OF CONGRESS CATALOGING-IN-PUBLICATION DATA}

\section{Elena Oumano}

Cinema today : a conversation with thirty-nine filmmakers from around the world / Elena Oumano.

p. $\mathrm{cm}$.

Filmmakers interviewed: Tomas Alfredson, Özcan Alper, Olivier Assayas, Serge Bozon, Catherine Breillat, Andrew Bujalski, Charles Burnett, Pedro Costa, Constantino CostaGravas, Claire Denis, Sergei Dvortsevoy, Jihan El-Tahri, Ari Folman, Matteo Garrone, Bette Gordon, Eric Guirado, Lance Hammer, Mia Hansen-Love, Mary Harron, Scott Hicks, Courtney Hunt, Agnès Jaoui, Kyoshi Kurosawa, Pablo Larrain, Anne Le Ny, Lucrecia Martel, Brillante Mendoza, Teona Strugar Mitevska, Gerardo Naranjo, Lucia Puenzo, Shamim Sarif, Paul Schrader, Céline Sciamma, Jerzy Skolimowski, Jean-Marie Téno, Ivo Trajkov, Melvin Van Peebles, Gary Winick, Jia Zhangke.

ISBN 978-0-8135-4876-0 (hardcover : alk. paper)-ISBN 978-0-8135-4877-7 (pbk. : alk. paper)

1. Motion picture producers and directors-Interviews. 2. Motion picturesProduction and direction. 3. Screenwriters-Interviews. 4. Motion pictures authorship. I. Oumano, Elena.

PN1998.2.C5675 2010

791.4302’320922-dc22 2009052307

A British Cataloging-in-Publication record for this book is available from the British Library.

Copyright @ 2011 by Elena Oumano

All rights reserved

No part of this book may be reproduced or utilized in any form or by any means, electronic or mechanical, or by any information storage and retrieval system, without written permission from the publisher. Please contact Rutgers University Press, 100 Joyce Kilmer Avenue, Piscataway, NJ 08854-8099. The only exception to this prohibition is "fair use" as defined by U.S. copyright law.

Visit our Web site: http://rutgerspress.rutgers.edu Manufactured in the United States of America 
For Lukas Alexander Roth and Sela Grace Roth 
